\title{
Gebären will gelernt sein! - Bildungsangebote zur Geburt zwischen emanzipatorischer Befähigung und neoliberaler Indienstnahme
}

\section{Lotte Rose}

Angenommen: 5. Januar 2022

(C) Der/die Autor(en) 2022

Zusammenfassung Ein Kind auf die Welt zu bringen, bedeutet heute, diese Aufgabe als Projekt des ,unternehmerischen Selbst“ zum eigenen Vorteil und Vorteil des Kindes selbstverantwortlich und erfolgreich zu managen. Erwartet wird nicht nur, gut informiert zu sein zu den Abläufen, Risiken und Interventionen bei der Geburt, sondern auch, eigene Wunschvorstellungen zur Geburt zu entwickeln, die eigene Gesundheit zu sichern, geburtsoptimierende Körpertechniken zu beherrschen und auf dem „Markt“ der Geburtshilfe die richtigen Entscheidungen zu treffen. Vor dem Hintergrund dieser Qualifikationsansprüche ist es für Schwangere und ihre Partner zum Standard geworden, Geburtsvorbereitungskurse zu besuchen. Der Beitrag rekonstruiert zunächst die Entwicklung der Geburt hin zu einer Bildungsaufgabe, um anschließend empirisch der Frage nachzugehen, was bei dieser Bildung genau passiert. Anhand ethnografischen Beobachtungsmaterials aus Bildungsangeboten ,rund um die Geburt“ wird nachgezeichnet, wie werdende Eltern von institutionellen Akteur_innen für die Geburt vorbereitet und qualifiziert werden, welche sozialen Anrufungen sie erleben, was öffentlich gemacht wird und was auch nicht. Nachgezeichnet wird schließlich auch, welche Geschlechterordnung der Geburt kreiert wird, indem der Vater in spezifischer und von der Mutter unterschiedener Weise als Bildungsobjekt adressiert wird. Abschließend werden die Befunde theoretisch vor dem Hintergrund gesellschaftlicher Neoliberalisierungen theoretisch reflektiert.

Schlüsselwörter Geburt · Natalität · Gender · Eltern

\footnotetext{
Availability of data Not applicable

Lotte Rose $(\bowtie)$

FB 4 Soziale Arbeit und Gesundheit, Frankfurt University of Applied Sciences,

Nibelungenplatz 1, 60318 Frankfurt am Main, Deutschland

E-Mail: rose@fb4.fra-uas.de
} 


\title{
Birth wants to be learned!-Educational opportunities for childbirth between emancipatory empowerment and neoliberal commissioning
}

\begin{abstract}
Bringing a child into the world today means managing this task as a project of the "entrepreneurial self" for the benefit and advantage of the child and mother. It is expected not only to be well informed about the processes, risks and interventions at birth, but also to develop one's own wishes about childbirth, to secure one's own health, to master birth-optimizing body techniques and to make the right decisions in the "market" of obstetrics. Against the background of these qualification requirements, it has become standard for pregnant women and their partners to attend birth preparation courses. The article first reconstructs the development of childbirth into an educational task, and then empirically investigates the question what happens exactly in this education. On the basis of ethnographic observational material from educational offers "around birth", it will be studied how expectant parents are prepared and qualified by institutional actors for childbirth, what social invocations they experience, what is made public and what is not. Finally, it will be shown which gender order of birth is created by addressing the father as an educational object in a specific way and different from the mother. Finally, the findings are theoretically reflected against the background of social neoliberalization.
\end{abstract}

Keywords Birth $\cdot$ Natality $\cdot$ Gender $\cdot$ Parents

Kinder auf die Welt zu bringen, ist eine simple anthropologisch-physiologische Konstante. Doch wie es stattfindet, ist eine höchst soziale Angelegenheit. Kennzeichnend für das Geburtskonzept der Gegenwart ist seine „Verschulung“. Die werdenden Eltern sind aufgefordert, Expertise für die Geburt zu entwickeln. Besonders prominent sind hierbei die Kurse zur Geburtsvorbereitung und Informationsabende in Kliniken. Doch was passiert genau an diesen historisch neuen und mittlerweile so alltäglichen Orten der elterlichen „Geburtsbildung“?

Der Beitrag zeichnet nach Ausführungen zum Wandel der Geburtskultur anhand ethnografischen Beobachtungsmaterials aus Informationsabenden und Bildungsangeboten zur Geburt nach, wie werdende Eltern von Institutionen für die Geburt vorbereitet werden. Dies wird abschließend theoretisch vor dem Hintergrund gesellschaftlicher Neoliberalisierungen eingeordnet.

\section{Historische Wandlungen des Geburtskonzeptes}

Im letzten Jahrhundert hat sich die Geburtspraxis tiefgreifend gewandelt. Im Kern geht es um eine zunehmende Selbstermächtigung der Gebärenden - dies wäre die emanzipatorische Lesart - oder aber um ihre zunehmende Responsibilisierung dies wäre die kritische und kulturpessimistische Lesart. Beide Modi sind miteinander verwoben und transformieren ,aus der Sache“ heraus die Geburt zu einem aufwendigen Bildungsgegenstand. 


\subsection{Geburt als Emanzipationsprojekt}

Bereits Anfang der 1980er-Jahre diagnostizierte Hardach-Pinke, dass Gebären ,weitgehend ein rationales, geplantes privates Unternehmen“ (1982, S. 204) geworden ist. Dies tat sie vor dem Hintergrund der sich in dieser Zeit etablierenden Ideen der selbstbestimmten und sanften Geburt, die die Geburtshilfe nachhaltig veränderten. Angetrieben wurde dies seit den 1970er-Jahren von medizinischen Geburtsreformern, aber auch durch die feministische Frauengesundheitsbewegung, die die klinische Geburtshilfe als einen Ort männlich-ärztlicher Dominanz kritisierte, die Frauen entmachtet (Oakley 1980; Davis-Floyd 1992; Metz-Becker 1997).

Die Ambivalenz des neuen natalen Selbstbestimmungsparadigmas zwischen Freiheit und Verantwortungsdruck tauchte in nachfolgenden Beiträgen immer wieder auf und wurde theoretisch weiterentwickelt (u. a. Beck und Beck-Gernsheim 1990; Rose 1993). So stellen Villa et al. (2011) fest, dass die Geburt „zu einem weiteren Projekt im Leben des „,unternehmerischen Selbst“““ geworden ist, das „ökonomisierten Logiken folgt" und effektiv zu managen ist (S. 12). Andere Autor_innen gehen noch weiter, wenn sie das Leistungsprinzip des Kinderkriegens exponieren. Heimerdinger (2012) klassifiziert die Praktiken prä- und postnataler Elternschaft als kompetitive Leistungsfelder, in denen ,sich der Wettbewerbsgedanke im Kontext der Elternschaft in weitaus umfassenderem und grundlegenderem Ausmaß breit gemacht hat, als es auf den ersten Blick scheinen mag" (Heimerdinger 2012, S. 265). Völkle und Muthmann stellen in ihrer Ethnografie fest, dass Geburt - und Stillen - als etwas gerahmt ist, „das von den (werdenden) Müttern zu leisten ist“ und dass an dieser Leistung ,bessere oder schlechtere Elternschaft“ bemessen wird (2019, S. 336). Für die ,natural birth“-Szene in Nordamerika, die die Geburt ohne ärztliche Eingriffe idealisiert, wird thematisiert, wie eine soziale Demarkationslinie zwischen Müttern mit „natürlicher“ oder „,medizinisch-assistierter“ Geburt entsteht und das Scheitern am Geburtsideal, das sich vor allem an der Nutzung der PDA-Narkose festmacht, zu einem sozialen Scheitern wird (Macdonald 2006; Rafalovich 2016).

Geburt hat nach diesen sozialwissenschaftlichen Diagnosen den Charakter des Schicksalhaften verloren. Sie ist nicht mehr eine Fügung „qua Natur“, auch nicht „qua Klinik“, die die Gebärende schlicht durchzustehen hat. Das moderne Narrativ zur Geburt ist stattdessen eines der emanzipatorischen Befreiung und Selbstverfügung, das auch generell für den gesamten Komplex des Kinderkriegens gilt. So zeichnen Heimerl und Hofmann (2016) anhand der Erzählungen von Eltern zu ihrem Elternwerden nach, wie stark das Bemühen ist, sich selbst als „Entscheider (und nicht als Entschiedene) " zu präsentieren (S. 426). Kinderkriegen darf nicht einfach so passieren, sondern ist Ergebnis von intensiven Auseinandersetzungen, nach denen „man sich schließlich bewusst für eine Schwangerschaft entscheidet" (ebd.). Dies gilt auch für die Geburt: sie darf auch nicht mehr einfach so passieren.

\subsection{Geburt als Bildungsprojekt - für Mütter und Väter}

Im Zuge dieser normativ-ideellen Veränderungen zur Geburt wird sie - historisch neu - als Bildungsgegenstand figuriert. Schließlich ist Expertise erforderlich, um die Geburt selbst in die Hand zu nehmen und zum eigenen Vorteil und zu dem des Kin- 
des gelungen zu meistern. Um sich entsprechend $\mathrm{zu}$ qualifizieren, wird von Eltern viel investiert: Sie recherchieren, lesen, lernen, üben, grübeln, entscheiden. Umfangreiche Ratgeberliteratur (vgl. Krumbügel 2015), Plattformen im Netz, medizinische Institutionen und Bildungsträger liefern das Wissen, um im „Markt“ der Geburtshilfe den eigenen Weg zu gehen. „Vielleicht sollte man, bevor man an Kinder zu denken wagt, heute besser ein Studium der Medizin absolvieren“, formulierte BeckGernsheim (1990, S. 152) zynisch vor vielen Jahren vor dem Hintergrund der natalen Qualifikationsansprüche. Weil es gilt, die Geburt als ,informed choice“ (Jung 2017, S. 31) selbst aktiv ,in die Hand zu nehmen“ und souverän zu steuern, müssen vor allem Mütter - aber auch Väter - diese Arbeit leisten. Zumindest müssen sie zeigen, dass sie willens dazu sind und sich darum bemühen. Dies wird besonders dann relevant, wenn es bei der Geburt - oder auch beim Stillen - zu Komplikationen kommt. In diesem Fall spielt für die moralische „Freisprechung“ der Mutter die Frage eine zentrale Rolle, ob sie alles Notwendige getan hat und willens war, es nicht so weit kommen zu lassen (Völkle und Muthmann 2019, S. 346).

Wenn Eltern sich für die anstehende Geburt bilden, entsprechen sie einerseits der zugewiesenen Selbstverantwortung, andererseits „empowern“ sie sich aber auch. Denn mit der aufgewandten Arbeit geht das Versprechen einher, die Chancen auf eine gute Geburt zu verbessern. „In den Anleitungsdispositiven des Stillens und Gebärens wird eine hohe Erwartung an das Gelingen einer ,natürlichen“ Geburt und des Stillens aufgebaut" (Völkle und Muthmann 2019, S. 340). Dies macht die „Verschulung“ der Geburt konsensfähig, akzeptabel und normal.

Bildung für eine gelingende Geburt bedeutet auch: Bildung als gemeinsames Paarprojekt. Dies ist eingelagert in die heterosexuelle Matrix der Familie. Es ist immer der Vater des Kindes, der als derjenige imaginiert und adressiert wird, der mit der Mutter die Passage zur Elternschaft aktiv zu bewerkstelligen hat. Dieses konservative Normalitätskonzept bestimmt die Bildungspraktiken im Feld der Natalität, aber ebenso die Forschungen. Geburtsvorbereitungskurse und Geburtshilfekliniken erwarten heterosexuelle Elternpaare, wie auch Forschungen sich auf diese kaprizieren. Dass Mütter alleinlebend sind, in einer gleichgeschlechtlichen Paarbeziehung leben oder auch mit einer ganz anderen Person in die Geburt gehen, bleibt praktisch und wissenschaftlich noch relativ unreflektiert.

Elaborierter ist das Wissen zur väterlichen Beteiligung an der Geburt. Die ForsaVäterstudie verweist auf $73 \%$ der Väter, die ihre Partnerin in den Kreißsaal begleiten (2013, S. 3), an anderer Stelle ist von 95\% die Rede (Süddeutsche 2014). Viele nehmen Urlaub, um das Wochenbett mit Frau und Kind zu teilen. Allerdings deutet einiges darauf hin, dass ihre Position an diesen Orten fragil ist.

Dies hat viel damit zu tun, dass Elternbildung aus der Tradition der Mütterschulen, die in den 1920er-Jahren gegründet wurden, entstanden ist. Erst Ende der 1960er wandelten sie sich zu Familienbildungsstätten mit gezielter Väterarbeit (Schirrmacher 2009, S. 37). Gleichwohl ist die Familienbildung weiterhin stark frauendominiert, sowohl was die Professionellen als auch die Nutzenden betrifft (vgl. Schäfer 2009). Väter stehen den Angeboten der Familienbildung distanzierter gegenüber, auch wenn sie für eine gleichberechtigte elterliche Arbeitsteilung plädieren (Neumann und Smolka 2016, S. 68). 
Dass es für Väter generell diffizil ist, sich in den Räumen des Kinderkriegens legitim Platz zu verschaffen, dafür liefert eine Studie zur Schwangerschaftsvorsorge Hinweise (Heimerl 2013): Väter werden bei den Untersuchungen übersehen, kaum angesprochen und nur als Zuschauer behandelt. Ähnliches wird für die Bildungsangebote ,rund um die Geburt“ - Geburtsvorbereitung, Säuglingspflege und -ernährung, Beikost - berichtet. Väter stehen auch hier am Rande, sind defensiv, werden übergangen, müssen Witze über sich ergehen lassen oder fehlen gänzlich (u. a. Rose und Pape 2020). Müller und Zillien (2016) arbeiten in ihrer ethnografischen Studie heraus, dass Geburtsvorbereitungskurse eine konservative Geschlechterordnung reorganisieren. Indem stets die „Naturwüchsigkeit einer primären Verantwortung der Mutter für ihr Kind“ exponiert wird, werden bereits vor der Geburt entscheidende „Pflöcke für eine geschlechterdifferenzierende Arbeitsaufteilung“ eingeschlagen, die auch postnatal weiterwirken (ebd., o. S.).

\section{Eine Ethnografie zur Geburtsvorbereitung}

Geburtsvorbereitungsangebote finden bei werdenden Eltern ein hohes Maß an Akzeptanz. (Lange und Ullrich 2018). Sie wurzeln in der Schwangerschaftsgymnastik, die sich in den 1950er-Jahren etablierte mit dem Ziel, durch Körperübungen und Aufklärung die Gesundheit der Schwangeren zu verbessern, Beschwerden zu lindern, aber auch den Geburtsverlauf zu erleichtern. Mit der feministischen Frauengesundheitsbewegung entstanden in den 1970ern dann geburtsvorbereitende Selbsthilfegruppen, die Schwangere ganzheitlich auf Geburt, Stillen und Wochenbett vorbereiteten. 1980 wird die „Gesellschaft für Geburtsvorbereitung“ gegründet, die Geburtsvorbereiterinnen ausbildet. Dies führt zur Etablierung der Geburtsvorbereitung als Bildungsprogramm für werdende Eltern für eine selbstbestimmte Geburt (Müller und Zillien 2016).

Die nachfolgenden Ausführungen zu den Vorgängen in Informations- und Bildungsangeboten zur Geburt basieren auf ethnografischen Daten aus einem Projektkomplex aus Feldforschungen an verschiedenen Bildungsorten zur Natalität. Es wurden teilnehmende Beobachtungen bei 23 Informationsabenden von Geburtseinrichtungen, zehn Geburtsvorbereitungs-, sieben Stillvorbereitungs-, zwei Säuglingspflegekursen, zehn Besuche in Geschäften zur Babyausstattung sowie in einem Geburtsvorbereitungskurs für Männer durchgeführt. ${ }^{1}$ Anliegen war, Formen impliziten sozialen Wissens einzufangen, die vor-sprachlich und vor-reflexiv sind und sich vor allem im praktisch-rituellen Handeln manifestieren (vgl. Kelle 2004, S. 637). Die Auswertung erfolgte in Anlehnung an die Grounded Theory (Strauss und Corbin 1996) in einer mehrphasigen Verschränkung von Datenerhebung und Datenauswertung. Der hier vorgelegte Beitrag bezieht sich auf die Empirie der Informationsabende und Bildungskurse zur Geburtsvorbereitung und Säuglingspflege.

\footnotetext{
1 Ein Großteil der teilnehmenden Beobachtungen wurde von Rhea Seehaus durchgeführt. An den Beobachtungen und Teilauswertungen waren zudem beteiligt: Judith Pape, Jörg Päplow, Kira Proll, Kerstin Schlesinger, Gloria Schmidt, Marc Schulz und Sonja Urban.
} 
Informationsabende zur Geburt werden von Geburtseinrichtungen als monatliche Abendveranstaltung angeboten. Ärzt_innen und/oder Hebammen und/oder Krankenschwestern informieren in Vorträgen zur Einrichtung, zu Geburtsabläufen und -möglichkeiten, Anmeldeprozeduren, aber auch zu Verhaltensempfehlungen vor allem für die Mutter. Oftmals folgt eine Führung durch den Kreißsaal, manchmal auch über die Wochenbettstation. Geburtsvorbereitungs- und Säuglingspflegekurse werden von Hebammenpraxen, Familienbildungsstätten, aber auch Geburtseinrichtungen durchgeführt. Sie sind für kleinere Gruppen angelegt und umfassen Wissensvermittlung, praktische Übungen und gemeinsamen Erfahrungsaustausch. Was passiert nun bei diesen Bildungsveranstaltungen? Was wird zum Thema gemacht, was nicht? Wie werden die werdenden Eltern dabei ,angerufen“ (Althusser 1977)?

\subsection{Vermeidung von Gesundheitsrisiken}

Zum Standard der Veranstaltungen gehört die Aufklärung zu Gesundheitsgefahren und Präventionsmaßnahmen in der Schwangerschaft. Dazu wird z. B. erläutert, was die Befunde im Mutterpass bedeuten. Damit wird den Vorsorgeuntersuchungen ,en passant“ Relevanz verliehen und die Teilnahme daran normalisiert. Es wird zudem medizinische Expertise vermittelt, die die Mutter befähigt, ihre persönlichen Untersuchungsdaten diagnostisch zu verstehen - eine Form der Emanzipation von gynäkologischer Wissensvormacht. Die Mutter wird zur medizinisch geschulten Selbstexpertin, die weiß, welche Erkrankungen drohen, woran sie zu erkennen sind und was sie dann tun muss.

Die Präventionsempfehlungen kreisen um die persönliche Lebensführung, vor allem um die Ernährung. Die Schwangeren werden aufgefordert, jetzt besonders auf eine gesunde Ernährung zu achten, nicht nur um die eigene Gesundheit zu sichern, sondern auch die des Fötus. Dies konstruiert eine existentiell-symbiotische Einheit von Mutter und Fötus. Was die Schwangere verspeist, ,verspeist“ auch der Fötus. Diese nutritive Einheit wird jedoch in den Kursen noch einmal besonders dramatisiert durch die Hinweise, dass Stoffe, deren Konsum für die Mutter harmlos sind, für den Fötus hochgefährlich sein können. Damit unterliegen Schwangere sehr viel strengeren Ernährungsregeln als nicht-Schwangere. Gleichzeitig wird sie als hochbedeutsamer Container des werdenden Lebens positioniert, deren eigenes Leben sich in der Relationalität zu ihrem Kind quasi auflöst.

Dagegen wird aber stellenweise auch aufbegehrt, wenn beispielsweise die verkündeten Speisetabus wieder infrage gestellt werden. Als es in einem Geburtsvorbereitungskurs um die Gesundheitsregeln geht, kommt es zu folgender Szene:

Abschließend berichtet die Kursleiterin Hannelore, dass so viel „hochgekocht“ würde, was alles nicht gegessen werden dürfte, da würde man die Leute noch ganz wahnsinnig mit machen. Sie würde auch viele „Ausländerinnen, Kroatinnen, Italienerinnen etc. “ betreuen, die würden damit alle ganz anders umgehen. Und sie wolle erst mal Studien sehen, ob es dort wirklich mehr Kinder mit „Schäden“ gebe. Ob denn hier alle auf alles verzichten würden, fragt sie etwas ungerichtet in die Runde. Carla ruft lachend aus: Sie sei Italienerin und natürlich würde sie Schinken essen, wenn er auf dem Tisch stünde. Wer könne 
denn da nein sagen, ruft sie laut aus. Viele lachen, auch Hannelore. Sie sagt, das müsse jeder für sich entscheiden, sie sei keine, die den Finger belehrend heben würde. Yvonne sagt in etwas mitleidiger Stimme, dass das Erste sei, was sie nach der Geburt essen würde: ein großes Baguette (sie malt mit den Fingern eine ansehnliche Größe in die Luft), belegt mit Serrano-Schinken und Salami. (Geburtsvorbereitungskurs im Geburtshaus)

Die Kursleiterin bezweifelt die Ernährungsempfehlungen. Ihre Einladung zur persönlichen Selbstoffenbarung der Teilnehmerinnen zum Umgang mit den Speisetabus wird in zwei Varianten beantwortet. Während Carla freimütig berichtet, dass sie die Regeln ignoriert, präsentiert sich Yvonne als Gefügige. Indem sie schildert, wie sie sich ihren Appetit auf das Wurstbaguette versagt, teilt sie mit, dass sie dem Tabu gehorcht, auch wenn es ihr nicht leichtfällt. Obwohl die Kursleiterin zuvor die Möglichkeit eröffnet hat, das quälende Speisetabu gefahrlos zu demontieren, nimmt die Protagonistin dieses Angebot nicht an, sondern sie reanimiert das herrschende Tabu als unhintergehbar. Dies spiegelt wider, wie sicher verankert einzelne Gesundheitsregeln bei den Schwangeren sind - so sicher, dass sie selbst die Provokationen durch die Fachautorität der Kursleitung überstehen.

Aufschlussreich ist das soziokulturelle „Doing Difference“ in dieser Szene. Eingeführt wird es durch die Kursleitung, die die Verhaltensweisen der Schwangeren aus anderen Ländern zitiert, um die herrschenden Speiseempfehlungen infrage zu stellen. Dies setzt sich dann fort in Carlas Rede. Das herrschende Nahrungstabu zu brechen, ist zwar erzählbar, muss aber legitimiert werden. Dies geschieht über das ethnisch-kulturelle „Anders-Sein“ der italienischen Herkunft.

\subsection{Natale Diagnosekompetenzen}

Alle Veranstaltungen vermitteln umfangreiche Informationen zum Schwangerschafts- und Geburtsverlauf, oftmals unterstützt durch Bildmaterial, das die einzelnen Etappen des körperlichen Veränderungsprozesses anhand von Zeichnungen der Körperinnenwelt schematisch typisiert abbildet. Ziel ist, die Schwangere zu befähigen, die eigene Körperwirklichkeit zu „erkennen“. Die Frauen werden aufgerufen, ihren Körper präzise zu beobachten, um Symptome sachgerecht zu diagnostizieren und die erforderlichen richtigen Schritte zu tun. Hierzu werden Körperphänomene besprochen, die Gesundheitsrisiken, vorzeitige Wehen, Senkwehen oder den Geburtsbeginn anzeigen.

Dem gegenüber stehen die Mitteilungen, dass diese Körperzeichen auch völlig belanglos - also kein Risikoindikator - sein können, oder dass sich die Schwangere auch von ihrem Gefühl leiten lassen soll, was die Bewertung ihrer Körperwahrnehmungen betrifft. Kristallisationspunkt dieser Doublebind-Botschaften sind die Blutungen der Schwangeren, wie exemplarisch bei diesem Vortrag einer Hebamme in einer Klinik:

Kommen solle man auch immer, wenn man Blutungen habe. Aber auch das sei nicht zwangsläufig ein Grund zur Besorgnis. In der Schwangerschaft könne 
man immer mal bluten. Aber wenn man „,so schmieren“ ${ }^{2}$ würde, auf der „Toilette nach dem Abputzen“ und vorher ,zwei Stunden spazieren war“, dann sollte man einfach die Füße hochlegen und einen Tee trinken. Wenn es aber nach zwei Stunden immer noch ,schmiert“, dann solle man kommen, oder wenn frisches, helles Blut dabei sei. (Informationsabend zur Geburt im Krankenhaus).

Die Informationen sind widersprüchlich: Die Blutung kann gefährlich sein, sie kann aber ebenso gut völlig banal sein. Die Schwangere erhält damit zwar diagnostisches Wissen, das aber in seiner Uneindeutigkeit vermutlich mehr irritiert als Handlungssicherheit erzeugt. Wenn die Blutung das eine und das andere bedeuten kann, bleibt die Handlungsentscheidung riskant.

\subsection{Wann beginnt die Geburt?}

Ein Schwerpunkt der diagnostischen „Ausbildung“ ist das Erkennen des Geburtsbeginns, um als Schwangere sich zur richtigen Zeit auf den Weg in die Klinik zu machen. Die ausführliche Thematisierung dieser Frage dramatisiert „unter der Hand“ das zeitliche „Richtig-Sein“. Es ist Aufgabe der Eltern, den Geburtsort weder zu früh, noch zu spät aufzusuchen. Die Erzählungen der Professionellen von Babys, die bei der Ankunft der Mutter in der Klinik schon fast „rausfallen“, weil man sich zu spät auf den Weg gemacht hat, gehören zum warnenden Standardrepertoire der Bildungsveranstaltungen.

Die Kursleiterin erzählt, dass alle wissen sollten, dass man Wehen an ganz verschiedenen Stellen des Körpers spüren könnte. Das sei wichtig zu wissen. Sie hätte schon eine Frau gehabt, die gedacht hätte, an einer Nierenkolik zu leiden. Dabei seien es Wehenschmerzen gewesen. Die hätte dann das Kind in der Dusche bekommen. Alle lachen, ein paar eher erstaunt-ungläubig. Und, führt sie die Geschichte weiter aus, weil sie dann einen Krankenwagen gerufen hätte, hätte sie noch mal mit dem Kind in die Klinik gemusst. Das sei, betont sie, blöd gewesen (Geburtsvorbereitungskurs im Geburtshaus).

Indem in abschätzig-ironisierendem Ton geschildert wird, welche unerfreulichen Folgen mütterliche ,Dummheit“ hat, werden die Zuhörerinnen aufgerufen, sich - anders als die Protagonistin der Geschichte - zu den Signalen der Geburt kundig zu machen. Die Schwangere, die die Wehenschmerzen falsch einschätzt, wird als Negativfolie vorgeführt, die die Anwesenden zur Abgrenzung einlädt. Man lacht, reagiert „erstaunt-ungläubig“ und markiert so, dass einem selbst dies nicht passieren wird.

Bei der Qualifizierung für das sachgerechte Erkennen des Geburtsbeginns spielen die Phänomene der Wehentätigkeit und des Blasensprungs ein prominente Rolle:

Die Hebamme sagt belustigt, dass es meistens so sei: „Das Telefon klingelt und der Vater sagt, was die Frau hat". Es wird klar, dass sie das nicht sonderlich begrüßt. Sie betont, dass Ferndiagnosen nicht möglich seien. Wenn die Frau

\footnotetext{
${ }^{2}$ Bei den hier und in den weiteren Protokollauszügen in Anführungszeichen gesetzten Textpassagen handelt es sich um Originalformulierungen der beobachteten Akteur_innen.
} 
ein ,bissl Schmerzen“ hätte, „ein bissl wie die Periode“ und wenn es dann auch noch im Rücken ziehen würde, so „fünf bis sechs Mal am Tag“, dann können „Sie mit Ihrem Partner noch in Ruhe essen gehen“. Wenn das aber über den ganzen Tag kommt, dann solle man vorbeikommen. „Zur Not schicken wir Sie wieder heim“. „Aber“, betont sie, ,schauen Sie, dass es eine Regelmäßigkeit hat!“" Anders sei es, wenn die Fruchtblase platzen würde, dann solle man immer kommen. Dabei gäbe es allerdings das „Problemchen“, dass man vorher klären sollte, ob das „Köpfchen“ fest im Becken wäre. Hier solle die Frau den Frauenarzt fragen. Wenn das nicht der Fall sei, dann solle ein „Liegendtransport“ erfolgen. [...] Ein falscher Alarm sei doch kein Problem. „Wenn Sie das innerliche Bedürfnis haben, das gibt es auch“, dann sei man hier auch richtig. „Das gibt's manchmal. Am Ende der Schwangerschaft spielen die Hormone ja manchmal verrückt.“ (Informationsabend zur Geburt im Krankenhaus).

Gleich zu Beginn wird von institutioneller Seite klargestellt, dass es allein in der elterlichen Verantwortung liegt zu klären, ob die Geburt begonnen hat. Der nachfolgende Text figuriert diese Verantwortung zwischen Schärfung und Lockerung von Leistungsansprüchen. Auf der einen Seite heißt es, dass die Schwangere in der Lage sein muss, korrekt zu entscheiden, wann sie in die Klinik muss und wann noch nicht. Dies ist verbunden mit der Mahnung, Indikatoren des Geburtsbeginns präzise zu prüfen, also alles dafür zu tun, dass es nicht zu Fehldiagnosen und Problemen kommt. Auf der anderen Seite wird der Leistungsdruck wieder zurückgenommen. So heißt es großzügig, dass das „falsche“ Erscheinen in der Geburtsklinik letztlich kein Problem ist und dass man auch kommen kann, wenn man schlicht das ,innerliche Bedürfnis" danach hat.

Die Diagnoseverantwortung der Schwangeren dehnt sich stellenweise auch dahingehend aus, dass sie Diagnosemöglichkeiten für die Klinik sichern muss, wie in dieser Szene sichtbar wird:

Man solle auch kommen, wenn mehr als ,ein Teelöffel“" Fruchtwasser abgehe. Das sei ja auch manchmal mit der Blase etwas schwierig mit dem dicken Bauch, aber es sei schon mehr Flüssigkeit. Die Ärztin wird sehr energisch: „Tragen Sie etwas Helles, unabhängig von der jeweiligen Mode, und bringen Sie das mit.“ Wegen der vermehrten Sekretion würden die meisten Frauen ja sowieso schon Slipeinlagen benutzen, man solle das „Höschen oder die Slipeinlage“ mitbringen, denn viele würden sich nach so etwas umziehen und ,wir haben dann eine trockene Frau und können den Blasensprung nicht mehr nachweisen“. Sie spricht das mit ernster Miene, es scheint wichtig, sie betont es später noch einmal. (Informationsabend zur Geburt im Krankenhaus).

Schwierig am Blasensprung scheint zu sein, diesen als solchen identifizieren und von einer harmlosen Schwangerschaftsinkontinenz unterscheiden zu können. Den Diagnosekompetenzen der Frauen wird offenbar nicht allzu sehr getraut, denn sie werden darüber aufgeklärt, was sie tun müssen, damit die Klinik selbst klären kann, ob es sich um einen Blasensprung handelt. Die Anweisungen zum Umgang mit den eigenen Slips haben infantilisierende und beschämende Momente. Während es in vielen anderen Szenen gerade darum geht, die Laienkompetenz der Frauen zu 
stärken, wird an dieser Stelle die medizinische Expertise für unerlässlich erklärt: Nur die klinische Institution kann - bei ausreichender Mithilfe der Frau - Urin vom Furchtwasser unterscheiden und so den Blasensprung diagnostizieren.

Die Qualifizierung der Schwangeren erweist sich damit als ein paradoxes Unterfangen. Sie muss sich zum einen bestmöglich Diagnosekompetenzen aneignen. Zum anderen stößt sie damit dennoch permanent an Grenzen, denn - so heißt es ja auch - die diagnostischen Indikatoren sind nicht sicher und eindeutig. So entsteht eine prinzipiell überbordende Sisyphos-Konstellation für die Schwangere.

Die dargestellten Vorgänge verweisen auch auf einen weiteren Umstand des Gebärens in der Gegenwart: die zunehmende Herauslösung der Schwangeren aus sozialen Kollektiven und ihre Vereinzelung. Den werdenden Müttern so umfangreiche Diagnosekompetenzen zu vermitteln, wird umso dringlicher, je weniger die Schwangere in ihrem Alltag von kundigen Menschen umgeben ist, die bei der Deutung der Körperzeichen sicher helfen und erforderliche Maßnahmen veranlassen können. Die Schwangere ist bei dieser Aufgabe auf sich allein gestellt und hat höchstens noch den Vater ihres Kindes dabei an ihrer Seite, der aber genaugenommen ebenso unkundig ist wie sie.

\subsection{Fitness für die Geburt}

Zum natalen Bildungscurriculum gehören auch Körperschulungen. Um die herausfordernde Geburtsarbeit gut durchzustehen, muss der Körper gesund, beweglich, kräftig und gleichzeitig entspannungsfähig sein. Sportliche Übungen, Massagen, Entspannungs- und Atemtechniken, die in den Kursen praktisch geübt und zu Hause weiter selbstständig praktiziert werden sollen, sollen dabei helfen.

In den Kursen trainieren die Schwangeren die Beckenbodenmuskulatur, sie dehnen ihre Leisten, lernen Techniken der Entspannung und der förderlichen Wehenatmung. Weitere Körpertechniken fokussieren ,,semi-medizinische“ Behandlungsmaßnahmen aus der traditionellen Erfahrungsmedizin und der Ernährung. Schwangeren wird beispielsweise für die letzten vier Schwangerschaftswochen ein spezieller Akupunkturplan oder auch Himbeerblättertee vorgeschlagen als Maßnahmen, um die Geburt schneller vonstattengehen zu lassen. In anderen Kursen wird den werdenden Müttern nahegelegt, regelmäßige Sitzbäder zu machen, um den Damm für die Strapazen der Geburt vorzubereiten. Die meisten dieser Tipps kreisen um das Versprechen einer schnelleren und schmerzarmeren Geburt.

In der folgenden Szene eines Geburtsvorbereitungskurses geht es um eine spezielle Schwangerschaftsdiät, die in einem Krankenhaus der Region den Schwangeren empfohlen wird. Dies führt dazu, dass sie auch in diesem Kurs zum Thema wird.

Als die Hebamme über Geburtserleichterungen spricht, bittet sie mich plötzlich, von der Empfehlung des Großkrankenhauses zu berichten, da ich das ja „durchexerziert“ hätte. ${ }^{3}$ Ich bin etwas überrumpelt, aber alle Augen sind be-

\footnotetext{
3 Da die Ethnografin selbst ein Jahr vorher einen Geburtsvorbereitungskurs bei dieser Hebamme besucht hatte, waren sie bereits vor der teilnehmenden Beobachtung miteinander bekannt. Aus diesem Zusammenhang wusste die Hebamme, dass die Ethnografin die vom Großkrankenhaus empfohlene Diät ausprobiert hatte.
} 
reits auf mich gerichtet. Also erkläre ich kurz den Zusammenhang zwischen einem hohen Blutzuckerspiegel und der verminderten Ausbildung von Prostaglandinrezeptoren im Gebärmutterhals (Im Nachhinein bin ich mir gar nicht mehr sicher, ob ich das korrekt dargestellt habe, aber die Hebamme korrigiert mich nicht.). Ich beschreibe die Empfehlung des Großkrankenhauses, deshalb die letzten Wochen vor der Geburt kein Weißmehl und keinen Zucker zu essen. Ich blicke in einige erstaunte, teilweise auch fassungslose Gesichter. Da ich merke, was das in der einen oder anderen auslösen könnte (zumal mir am Vortag bereits der Süßigkeitenkonsum der Schwangeren aufgefallen war), sage ich noch, dass ich natürlich nicht wüsste, ob es wirklich helfen würde, und man sicher selbst entscheiden müsse, ob einem das guttue. Aber die Hebamme erklärt, dass sie auch den Eindruck gehabt hätte, dass eine Geburt schneller verlaufen würde. Sie selbst würde es auf jeden Fall machen. Was man hätte, das hätte man, schaden würde es sicher nicht. [...] Christoph beugt sich vor und angelt sich eine Süßigkeit aus dem Kreis. Er bietet sie Meike an. Sie guckt ihn mit einem wehleidigen Blick an und schüttelt langsam dem Kopf. Ich frage mich, ob sie die Süßigkeit aufgrund meiner Ausführungen nicht isst, denn gestern aß sie durchaus von den angebotenen Süßigkeiten. (Geburtsvorbereitungskurs Geburtshaus)

Die Schwangeren werden zur Geburtsoptimierung zum Lustverzicht angehalten. Damit wird ihnen Kontrollmacht über den Geburtsprozess verliehen: Wenn sie sich an die empfohlene Diät halten, werden sie eine leichte Geburt haben. Die ängstigenden Realitäten der Geburt - ihre Schmerzen, ihre lange Dauer - werden als etwas konstruiert, dem durch eigenes Tun aus eigener Kraft zu entkommen ist - so das magische Versprechen.

Die anwesenden Frauen reagieren so betroffen auf das Diät-Narrativ, dass die Ethnografin in die Bredouille gerät. Sie will den getätigten Responsibilisierungsaufruf an die Schwangeren wieder zurücknehmen, kann dies aber nicht. Ihre Bemühungen, die Diät zu relativieren, reproduziert erneut Responsibilisierungen. Indem sie betont, dass jede Frau selbst entscheiden müsse, was sie von dem Tipp hält, verortet sie die Lösung des aufgebrochenen Diät-Dilemmas bei der Schwangeren selbst. Die Verheißung der Diät kann nicht mehr ungesagt gemacht werden. Einmal in der Welt, können die problematisierten Lebensmittel nicht mehr gedankenlos wie noch tags zuvor verzehrt werden - wie abschließend bei Meike zu sehen ist. Die Schwangeren können nicht mehr anders, als sich individuell und aktiv dazu zu verhalten.

\subsection{Wissen, was man will}

Ein weiterer Qualifizierungsauftrag an die Schwangere ist die Entwicklung eines persönlichen Geburtskonzepts. Dies umfasst Entscheidungen zum Geburtsort, zur Begleitung, Gebärhaltung, zu Gebärutensilien, Schmerzmitteln und anderweitigen medizinischen Interventionen, Tätigkeiten der Hebamme, des Partners, Musik, Raumund Badewasserdüfte, Nachgeburt und manches mehr. Eine solche Planung setzt eine gut informierte Schwangere voraus, die das Arsenal möglicher Requisiten und Praktiken der Geburtssituation nicht nur kennt, sondern sie für sich abgewogen und 
ausgewählt hat. Sie erzeugt die Gewissheit, dass eine Geburt planbar ist. Die Gebärende wird damit immer wieder demonstrativ zur verantwortlichen Regisseurin des Geburtsgeschehen erklärt wie z.B. in diesem Fall:

Während der Kreissaalführung kommt die Hebamme auf ihre Arbeit zu sprechen. Eine gute Geburt sei eine solche, erläutert sie, bei der die Hebamme nichts machen, sondern nur dabei sei und staunen würde. Hebammen würden immer dann Angebote machen, wenn die Frauen das möchten. (Informationsabend zur Geburt im Krankenhaus)

Die Botschaft ist: Die ideale Geburt ist, wenn die Schwangere sich auf ihre Regieposition erfolgreich vorbereitet hat und diese überzeugend einnimmt, weil sie weiß, was sie will, kann, braucht und was sie nicht will, nicht kann und nicht braucht. Diese Subjektanrufungen der Frauen als eigenverantwortliche Geburtsgestalterinnen gehen oftmals mit der Präsentation einer „Negativfolie“ einher, wie der Fortgang der Szene zeigt.

Es würde auch immer welche geben, die nicht wüssten, was sie wollten. Die würden dann unter der Geburt so dastehen und Wehen veratmen: Die Hebamme klammert sich mit beiden Händen hinter ihrem Rücken an den Heizkörper, fällt ins Hohlkreuz, streckt das Becken nach vorne, wirft den Kopf nach hinten und schnauft schwer. Das sei nicht gut, sagt sie, als sie die Pantomime abbricht, denn das Kind müsse ins Becken und würde so nicht ins Becken kommen. Sie schmunzelt, wirft aber auch einen intensiven Blick in die Runde der ZuhörerInnen, so als solle das allen klarwerden. Sie würde dann in der Regel fragen, was die Frauen möchten. Manche würden dann jedoch sagen, dass sie das nicht wüssten. Sie imitiert eine Frau mit weinerlicher, aufgelöster Stimme. Dann würde sie fragen, ob die Frau in die Wanne wolle. Die würde dann manchmal völlig entsetzt sein, dann würde sie fragen, ob sie noch ein bisschen laufen wolle. Wenn die Frau dann sage ,zu anstrengend“ (wieder die weinerliche Stimme), dann sage sie der Frau, dass es nur noch zwei Möglichkeiten gäbe: Entweder im „Bett einkuscheln oder auf den Pezziball“. Sie strahlt die Anwesenden an, so könne man auch nach dem Ausschlussprinzip arbeiten. (Informationsabend zur Geburt im Krankenhaus)

Die detaillierte Schilderung einer unvorbereiteten Schwangeren, die weder weiß, was sie zu tun hat, noch weiß, was sie will, hat eine distinktive Erziehungsfunktion: So wie diese Frau sollen sich die anwesenden Zuhörerinnen nicht verhalten, denn damit erschweren sie die Geburt und die effektive Kooperation mit der Hebamme. Unkundig zu Geburt zu sein, wird in dieser Inszenierung zum persönlichen Makel und nicht zum Anlass mitfühlender Sorge. Zwar kümmert sich die Hebamme auch um solche Fälle, aber der Subtext ist, dass dies eine Zumutung für die Professionellen ist.

Gleichwohl fehlt in den Kursen nicht der Hinweis darauf, dass unter der Geburt auch Situationen entstehen können, in denen sich die Gebärende dem fügen muss, was klinische Expert_innen ,zu ihrem Besten“ veranlassen, wenn es zu medizinischen Problemen kommt. Solche Hinweise erfolgen in der Regel in bagatellisierendem Duktus, der die Fremdverfügung bei der Geburt als unvermeidbare und nicht 
hinterfragbare Selbstverständlichkeit konstruiert. Damit sieht sich die Schwangere disparaten Botschaften ausgesetzt: Sie wird aufgefordert, sich zum autonomen Subjekt der Geburt zu machen, soll aber gleichzeitig flexibel bleiben und sich gegebenenfalls umstellen und die Position der fremdbestimmten Patientin einnehmen.

Besondere Prominenz in der Geburtsplanung hat die Wahl der Gebärposition. Routinemäßig wird darauf hingewiesen, dass die Rückenlage als ungeeignet gilt. Dies ist häufig verbunden mit drastischen Diffamierungen. So erklärt eine Hebamme die Rückenlage als die ,schrecklichste, beschissenste, schlechteste Geburtsposition nach dem Kopfstand“. Offensiv werden stattdessen alternative Gebärpositionen und dazu notwendige Instrumentarien wie Seile, Wanne oder Hocker vorgestellt. Ebenso werden die Schwangeren eingeladen, verschiedene Positionen selbst auszuprobieren, und es werden Tipps zur Optimierung der Körperhaltungen gegeben. Zudem werden die Frauen regelmäßig dazu aufgerufen, sich dagegen zu verwehren, wenn sie im Kreißsaal vom Personal zum Gebären in Rückenlage aufgefordert werden.

Die historisch neue Optionalität der Gebärposition (vgl. Tews 2007) weist der Gebärenden automatisch auch eine neue Bildungsaufgabe zu, denn sie muss sich mit den unterschiedlichen Möglichkeiten auseinandersetzen und klären, welche Haltung sie favorisiert. Zugleich darf sie sich nicht mehr ,einfach“ auf den Rücken legen: Diese Gebärposition wird im gesamten Protokollmaterial abgewertet. Betrachtet man jedoch die materiale Ausstattung der Kreißsäle, ist dies paradox. Zentrales Mobiliar sämtlicher Kreißsäle der ethnografischen Feldforschung war das Gebärbett, während andere Gebärutensilien räumlich marginalisiert waren. Sie standen am Rande, waren verborgen in Schränken, mussten erst geholt werden, wenn die Sprache darauf kam. Was die Schwangere im Kurs lernt, wird in den klinischen Praxisroutinen sabotiert.

\subsection{Was tut der Vater bei der Geburt?}

In den Bildungsangeboten erhält auch der Vater Lernaufgaben für die Geburt. Seine bedeutsamste Funktion ist der Schutz und die Interessenssicherung seiner Frau unter der Geburt. In den Kursen wird stets angemahnt, dass die Schwangeren ihren Partner über ihre Geburtswünsche unterrichten sollen, damit die Partner diese im Kreißsaal anwaltschaftlich vertreten können, wenn die Gebärende sie angesichts des Geburtsstresses vergisst oder sie dazu keine Kraft mehr hat. In einem Geburtsvorbereitungskurs wird dies von der Leiterin angesprochen:

Es könne auch sein, dass die Frau Schmerzen hätte, der Wehenschreiber das jedoch nicht anzeigen würde. [...] Wenn da eine Äußerung käme wie „das kann nicht sein“, wäre das eine unsensible Geburtsbegleitung. Die Väter sollten aufpassen, dass so etwas nicht ,wegdiskutiert“ würde. Sie sollten sich auf die Seite der Frau schlagen, nicht auf die der Hebamme. Caro murmelt trocken: „Mit der Frau geht man heim. “Es gibt großes Gelächter. Die Kursleiterin wird eindringlich: Die Väter hätten eine „Dolmetscherfunktion, ihr könnt am Gesichtsausdruck der Frau sehen, wie es den Frauen geht. Die Hebamme kann das nicht.“ (Geburtsvorbereitungskurs einer Familienbildungsstätte)

Der Vater wird angerufen als ritterlicher Beschützer seiner Frau. Um diese Aufgabe zu plausibilisieren, wird die Institution des Kreißsaales als feindselig präsentiert. 
Dies geht so weit, dass selbst die Hebamme, die ansonsten oftmals als die „Schutzherrin" der Gebärenden gilt, in diesem Fall zur Widersacherin gemacht wird. Weil - so heißt es - im Kreißsaal die Gebärende missachtet wird, muss sich der Vater wachsam vor seine entkräftete Frau stellen und standhaft um ihr Wohlergehen kämpfen.

Zudem ist der Vater als allgemeiner Wohltäter seiner Frau gefragt. Er muss in der Lage sein, Qual und Pein des Gebärens zu lindern. Das Repertoire der vermittelten Praktiken reicht von Essens- und Erfrischungsangeboten über körperunterstützende Handgriffe beim Baden, Maßnahmen der Schmerzlinderung und praktischen Zugriffen in der Austreibungsphase bis hin zu emotionalem Zuspruch. Väter werden aufgefordert, wie die Schwangeren auch die Technik der Wehenatmung zu erlernen, um die Gebärende darin unterstützen können, wenn die Kraft dazu nachlässt. Regelhaft erfolgt zudem eine Einweisung in Massagetechniken zur Reduktion der Geburtsschmerzen, die im Kurs praktisch an den Körpern der eigenen Frauen geübt werden.

Alle Körperübungen werden ausnahmslos von den jeweiligen Elternpaaren miteinander absolviert, niemals kommt es zu Vermischungen zwischen den Paaren. Nur wenn Frauen ohne Begleitung kommen, kann es zu Abweichungen kommen. So wird eine Ethnografin in einem Kurs von der Leiterin aufgefordert, bei der Massageübung die einzige Frau, die allein gekommen ist, zu massieren.

Dann fragt sie mich, ob ich die Übung nicht mit Carla machen könnte. Ich bin am Schreiben und merke erst gar nicht, dass sie mich meint. Aber sie spricht mich noch mal an. Ich traue mich nicht so recht nein zu sagen, weil ich nicht das Gefühl vermitteln möchte, dass ich Carla unangenehm finde. Also sage ich zu. (Geburtsvorbereitungskurs im Geburtshaus)

Die soziale Irritation der Ethnografin angesichts der Aufforderung, sich als Übungspartnerin für eine Kursteilnehmerin zur Verfügung zu stellen, spiegelt die heterosexuelle Matrix der Kurse wider. Zentrales Lernsubjekt ist das intime Paar, das miteinander arbeitet. Berührungen sind nur paarintern üblich. So sind die Kursgruppen durch starke räumliche Segmentierungen der jeweiligen Elternpaare und Inszenierungen von intimer Nähe gekennzeichnet: jedes Paar sitzt nah beieinander, berührt sich während des Kursprogramms immer wieder, lehnt sich aneinander oder tauscht zärtlich Gesten aus.

Tom beginnt, Marions Bauch mit der flachen Hand zu streicheln. Dann schiebt er ihren Pulli hoch. Darunter trägt sie eine weitere Lage Stoff, auf dieser streichelt er weiter, schiebt diese aber [...] etwas nach oben. Nun liegt seine Hand auf dem Bund ihres Rockes. Dort streichelt er weiter. Sie hört währenddessen aufmerksam der Kursleiterin zu. (Geburtsvorbereitungskurs einer Familienbildungsstätte)

Diese und ähnliche Gesten der Nähe, die in anderweitigen öffentlichen Erwachsenen-Räumen verpönt wären, sind in den Geburtsvorbereitungskursen erlaubt. Dass die Kursräume in dieser Weise zu Bühnen der symbolischen Inszenierung elterlicher Verbundenheit werden, lässt sich lesen als Ausdruck des modernen Konstrukts zum Kinderkriegen. Es ist das Projekt des intim verbundenen heterosexuellen Paa- 
res, Symbol seiner Innigkeit. Schließlich soll die Geburt - und die nachfolgende Kinderpflege - die Arbeit beider Elternteile und nicht nur die der Mutter sein. Die Elternbildung zur Geburt offenbart sich damit als Normalisierungsakteur einer spezifischen paternalen Ordnung. Das heterosexuelle Paar wird als Subjekt der Geburt des eigenen Kindes adressiert, exponiert und aneinandergebunden und von anderen sozialen Entitäten abgesondert.

Zugleich wird eine spezifische Ordnung zwischen Vater und Mutter hervorgebracht und normalisiert: Der Vater als „Dienstleister“ für die Mutter. Während die Mutter Techniken der Selbstoptimierung erlernt, geht es für den Vater darum, sich dafür zu qualifizieren, das Tun seiner Frau zu optimieren. Seine Bildungsbedarfe zum Geburtsgeschehen sind primär über die Hilfeerfordernisse der Gebärenden definiert. Dies wird besonders deutlich sichtbar in den exklusiven Bildungsangeboten für werdende Väter. ${ }^{4}$

Auch wenn diese immer als Raum zur Beschäftigung mit den eigenen Themen der Väter propagiert werden, zeigt sich in der Praxis, dass im Grunde alle Vorgänge auf ,die Frau“ verwiesen sind. Sowohl Inputs und Gesprächsanimationen der männlichen Kursleiter als auch Fragen und Beiträge der anwesenden Männer kreisen um das, was bei der eigenen Frau in der Schwangerschaft und unter der Geburt passiert und wie man als Vater damit sachgerecht umgeht. Dabei kristallisiert sich jedoch ein Thema heraus, das ausschließlich in den „Väter-Räumen“ bearbeitet wird: Sexualität. So kommt es in einem Vater-Kurs gleich zu Beginn zu folgender Szene: Der Kursleiter stellt das Kursprogramm vor und kündigt an, dass zum Abschluss noch eine Hebamme dazukommen wird.

„Bis dahin haben wir die Zeit für uns, um über die Fragen zu sprechen, die wir Männer haben. Auch für das Thema Sexualität in der Schwangerschaft ... dafür ist Raum“, leitet der Kursleiter die folgende Gesprächsrunde ein.

Sexualität wird in diesem Skript als männliche Differenzfigur markiert. Tatsächlich erlebten wir kein Angebot, an dem werdende Mütter teilnahmen, an dem dieses Thema platziert wurde. Dies folgt vordergründig der geschlechtersymbolischen Codierung von Sexualität. Hintergründig verweist es aber auch auf den Bedarf einer solchen Differenzmarkierung. Während ansonsten die Elternbildungsprogramme zur Geburt stark von dem Bemühen gekennzeichnet sind, Differenzen oder gar Konflikten zwischen Vater und Mutter keinen Raum zu geben, sondern erfolgreich das Gemeinsame zu inszenieren, scheint Sexualität ein - und vielleicht auch der einzige Gegenstand zu sein, an dem offenbar in öffentlich legitimer Weise Interessengegensätze zwischen Vater und Mutter und „väterliche Probleme“ artikuliert werden können.

\footnotetext{
${ }^{4}$ Im Untersuchungssample waren nur zwei entsprechende Veranstaltungen - ein Vaterkurs und eine Kurseinheit in einem Elternkurs, in dem Väter und Mütter zeitweise in geschlechtshomogenen Gruppen unter der Leitung einer gleichgeschlechtlichen Person getrennt arbeiteten. Dennoch lassen die Beobachtungen hier aufschlussreiche Rückschlüsse zum Doing Gender in der natalitätsbezogenen Elternbildung zu.
} 


\section{Abschluss: Geburtsbildung zwischen Befähigung und neoliberaler Indienstnahme}

Die Ethnografie zu den pränatalen Bildungsangeboten zeigt, dass und wie sich Geburt vom schicksalhaften biografischen Krisenereignis, dem die Gebärende ausgeliefert ist, $\mathrm{zu}$ einem emanzipatorischen Bildungsgegenstand gewandelt hat, der die Gebärende - mit dem Vater des Kindes - als gestaltendes Subjekt der Geburt positioniert. Die Vermittlung von medizinischem Wissen zu Schwangerschaft und Geburt, zu Risiken und Prävention, die Aufklärung zu den institutionellen Abläufen in der Klinik und ihre Besichtigung und schließlich die praktische Einweisung in Techniken der Geburtsvorbereitung, -erleichterung und Schmerzlinderung und deren Übung soll das werdende Elternpaar dazu befähigen, die Geburt des eigenen Kindes zum einen selbstbestimmt in die Hand zu nehmen, zum anderen aber auch Geburtsqualen für die Mutter zu minimieren und dem Kind einen möglichst reibungslosen Start ins Leben zu ermöglichen.

Die rekonstruierten Bildungspraktiken zur Geburt lassen sich somit unschwer als Element moderner ,Subjektivierungsprogramme“ (Bröckling 2002, S. 178) identifizieren, deren Ziel ist, die gesamte „Lebensführung auf unternehmerisches Handeln“ (ebd., S. 179) auszurichten. Was für alle Individuen gilt, gilt auch für die Schwangere. Sie ist aufgefordert, „Arbeit an sich“ (Rieger 2002, S. 79) selbst zu vollbringen, damit das „Unternehmen Geburt“ gut gelingt. Dies lässt sich mit Bezug auf Nina Oelkers (2007, S. 407) als spezifische Form der Responsibilisierung verstehen.

Werdende Eltern müssen, um Anerkennung zu finden, verantwortliche Elternschaft unter Beweis stellen. Dies tun sie nicht allein mit der Inanspruchnahme ärztlicher Vorsorgeuntersuchungen, der Erfüllung der Anweisungen zur Ernährung und Lebensweise (Ruhl 1999), sondern auch mit ihren Bildungsbemühungen. Wer dies nicht tut, das lehren die Bildungsangebote, erfährt keine Empathie, was ja im Prinzip auch denkbar wäre, sondern wird diskreditiert, der Lächerlichkeit anheimgegeben und als „Abschreckungsgeschichte“ funktionalisiert. Er dient dazu, unverantwortliche Elternschaft für die Anwesenden zu illustrieren und Distinktion zu aktivieren, wie anhand einzelner ethnografischer Sequenzen gezeigt wurde.

Verantwortung für die Geburt muss auch der Vater übernehmen. Die Ethnografie belegt, dass Väter nicht nur wie die Mütter Bildungsangebote aufsuchen, sondern dass sie aktiv als Lernende gefordert sind. Auch wenn auf diese Weise die Geburt als „Frauensache“ demontiert wird und das Elternpaar in der natalen Statuspassage eng aneinandergebunden und scheinbar gleich gemacht wird, organisiert sich darin gleichzeitig eine traditionell-binäre Geschlechterordnung. Als die „,normalen“ paternalen Kooperationspartner_innen der Mütter gelten in den Diskursen und Praktiken der Geburtsbildung letztlich nur Väter des kommenden Kindes. Anderweitige soziale Beziehungskonstellationen haben keinen sozial anerkannten Platz. Darüber hinaus sind die Bildungsinhalte, folgt man den ethnografischen Einblicken, primär auf das ausgerichtet, was bei der Gebärenden vor sich geht. Der Vater wird ausschließlich in Relationalität zur Mutter als ihr helfender Begleiter adressiert, hat keine Ansprüche auf eigene Bildungsbedarfe - abgesehen von seiner Sexualität mit einer schwangeren oder entbundenen Frau. 
Geburt ist damit weniger als „Care-Anlass“ konzipiert, der die Gebärende vorrausetzungslos berechtigt, gesellschaftliche Fürsorge beanspruchen (Jung 2017, S. 43) und sich in der Krise anderen anvertrauen zu können, die sich bestmöglich zu ihrem und des Kindes Wohl um sie kümmern. Schwanger zu sein und zu gebären erlaubt nicht, die Position der Hilfe- und Fürsorgebedürftigen, auch nicht der Verängstigten und Überforderten und schon gar nicht der Desinteressierten oder ,Bildungsverweigerin“ einnehmen zu können, sondern es ruft dazu auf, sich selbst bestmöglich zu wappnen für das, was ansteht.

Wenn so viele Eltern selbstverständlich Geburtsvorbereitungskurse und Informationsabende besuchen, wenn sie sich dort durchgängig lernwillig und wissbegierig zeigen, spiegelt dies die normalisierte Identifizierung mit dem Konzept der Emanzipation durch Bildung wider. Erinnern wir uns an die ethnografischen Szenen: Niemals war Aufbegehren gegen die Bildungserwartungen vonseiten der Teilnehmenden zu beobachten, immer zeigten sie sich kooperativ gegenüber den Lehrkräften.

Die Empirie offenbart schließlich auch einen charakteristischen Double-bindCharakter der Bildungspraktiken. Die Geburt wird ,als einerseits natürlicherweise funktionierend“ und ,andererseits als zu erbringende" Leistung gerahmt (Völkle und Muthmann 2019, S. 340). In den Kursen werden Körpersymptome der Schwangerschaft dramatisiert und dann bagatellisiert. Es werden Empfehlungen zur Risikoprävention gegeben, die wieder infrage gestellt werden. Es wird zu Wachsamkeit und Vorbereitung geraten, aber auch das Vertrauen in das Gelingen natürlicher Vorgänge und der Körper-Intuition propagiert. Zudem greifen Ermächtigung und Entmächtigung stetig ineinander. Die Eltern werden angerufen als selbstbestimmte Subjekte, die sich für die gelingende Geburt befähigen können und müssen. Gleichzeitig wird aber präsent gehalten, dass alles auch ganz anders laufen kann und die Geburt am Ende entgegen der Versprechen und Hoffnungen ,entgleitet“ und von der Klinik übernommen werden muss - dann nämlich, wenn es zu fatalen Geburtskomplikationen kommt oder die Mutter sich als unfähig zur Selbstbestimmung und produktiven Mitarbeit erweisen sollte. Diese widersprüchlichen Narrative haben ein gewisses „Verwirrungspotenzial“" für werdende Eltern.

Gleichwohl ist es bei genauerem Blick auch funktional für das ökonomisierte, moderne Gesundheitswesen, denn Eltern werden zugleich zur natalen Selbstqualifizierung und -steuerung und Unterordnung unter klinische Routinen angerufen. Mit Bezug auf die Studie von Regina Dackweiler zur Geschichte der Frauengesundheitsbewegung (2010, S. 147) offenbart sich, wie die Idee der Selbstbestimmung, die für die feministische Kritik am Gesundheitswesen und der Geburtshilfe so prominent war, an das Regime neoliberaler Selbstsorge anschlussfähig geworden ist, in dem Lebensrisiken von den Individuen selbst bewältigt werden müssen. Denn die Aufforderung zur Selbstverantwortung der Geburt korrespondiert in idealer Weise mit einer wohlfahrtsstaatlichen Politik, in der die öffentliche Gesundheitssorge betriebswirtschaftlich ,effektiviert“ wird. Sie ist zu einem wettbewerbsorientierten Dienstleistungsmarkt transformiert worden, in dem aus Patient_innen anspruchsvolle Kund_innen geworden sind (vgl. Dierks et al. 2001, S. 14), die sich aber nun auch um viel mehr selbst kümmern müssen. Werdende Eltern müssen sich auf dem „Geburtshilfemarkt" kundig machen, urteils- und entscheidungsfähig werden, um bestmöglich zu profitieren. 
Dies schließt an die Forschungen von Tina Jung an, die deutlich machen, wie mit der Verschlechterung der Versorgungsqualität in der Geburtshilfe gleichzeitig die Selbstverfügung der Gebärenden popularisiert wird (Jung 2017, S. 31). Je weniger Ressourcen für die Versorgung der Gebärenden bereitgestellt werden, desto deutlicher bedarf es einer gut gebildeten Gebärenden, die weiß, was sie zu tun hat. Auch der Einzug der Väter in die Kreißsäle und ihre geburtsvorbereitende „Beschulung“ offenbart sich damit als etwas, bei dem es um sehr viel mehr geht als die Förderung ,neuer Väterlichkeit“. Der Gebärenden steht damit in der Krise ein Mensch zur Seite, der klinisch nicht bereitgestellt wird. Der geburtsbegleitende Vater ist also institutionell hochgradig ,,verzweckt“. Ignoriert wird dabei, dass dieser Helfer sich selbst in einer Krise befindet.

Was ideell als emanzipatorische Chance zur Sicherung von Autonomie beim Gebären erscheint, offenbart sich damit janusköpfig. Ein desolater Geburtsverlauf mit möglicherweise auch krisenhaftem Ausgang ist nicht mehr wahrnehmbar und begreifbar als Ergebnis schlechter struktureller, materieller und personeller Sorge oder auch eines schicksalhaften Körpers, sondern erscheint nur noch als logische Folge unzureichender und selbstverschuldeter Vorbereitung der Gebärenden selbst (Jung 2017, S. 43).

$\mathrm{Zu}$ guter Letzt erweist sich die „Verschulung“ der Geburt als Reproduktionsort sozialer Ungleichheiten. Soweit sich dies in unserer Studie ethnografisch bestimmen ließ, waren es Eltern aus Bildungsmilieus, die an den von uns untersuchten Bildungsangeboten zur Geburt teilnahmen. Dies entspricht den Befunden diverser Untersuchungen, nach denen die pränatalen Präventions- und Bildungsmaßnahmen nicht von allen sozialen Gruppen in gleicher Weise genutzt werden. Bereits in den 1990ern wurde problematisiert, dass Frauen aus der Mittelschicht am häufigsten teilnehmen, junge, unverheiratete Frauen aus der Arbeiterklasse am seltensten (Cliff und Deery 1996). Diese Klassendifferenz wurde im weiteren Verlauf immer wieder bestätigt (Krahl 2012; Lange und Ullrich 2018). Schwarz und Schücking (2004) stellen für Deutschland fest, dass die am besten informierten und gesündesten Frauen überversorgt sind, während die risikoexponierteren Frauen in prekären Lebensverhältnissen nur teilweise von den Vorsorgemaßnahmen erreicht werden. So ist empirisch auch nachweisbar, dass Migrantinnen der ersten Generation, Frauen mit geringen Deutschkenntnissen, Frauen mit Fluchterfahrungen und unsicherem Aufenthaltsstatus und Drogenkonsumierende seltener die vorgeburtlichen Vorsorgeangebote nutzen (Rose und Planitz 2021).

Dies zeigt: Nicht alle Bevölkerungsgruppen aktiviert der natale Optimierungsund Bildungsimperativ in gleicher Weise, nicht alle haben die gleichen Chancen, von der vorhandenen Bildungsinfrastruktur zu profitieren. Wie und warum Menschen in prekären Lebenslagen ausgeschlossen werden und welche Folgen dies für die Geburtspraktiken und die soziale Positionierung im Gesundheitswesen wie auch im gesellschaftlichen Raum hat, dazu liegt derzeit nur wenig Wissen vor. In der Familien- und Natalitätsforschung wird mittlerweile zumindest selbstkritisch die starke Mittelschichtsfokussierung problematisiert (u. a. Lange und Ullrich 2018; Villa et al. 2011), ohne dass sich jedoch Veränderungen bislang abzeichnen. Hier deuten sich also offene Forschungsbedarfe für die Zukunft an. 
Danksagung Ich danke Dr. Rhea Seehaus für die langjährigen gemeinsamen Forschungstätigkeiten ,rund um die Geburt", die bereichernden Diskussionen und insbesondere für ihre aufwendigen Feldforschungen und kompetenten Auswertungen, ohne die diese Publikation nicht möglich gewesen wäre.

Funding Die empirischen Datenerhebungen, die für den Beitrag genutzt wurden, wurde vom Hessischen Ministerium für Wissenschaft und Kunst und vom Gender- und Frauenforschungszentrum der Hessischen Hochschulen (gFFZ) gefördert.

Funding Open Access funding enabled and organized by Projekt DEAL.

Open Access Dieser Artikel wird unter der Creative Commons Namensnennung 4.0 International Lizenz veröffentlicht, welche die Nutzung, Vervielfältigung, Bearbeitung, Verbreitung und Wiedergabe in jeglichem Medium und Format erlaubt, sofern Sie den/die ursprünglichen Autor(en) und die Quelle ordnungsgemäß nennen, einen Link zur Creative Commons Lizenz beifügen und angeben, ob Änderungen vorgenommen wurden.

Die in diesem Artikel enthaltenen Bilder und sonstiges Drittmaterial unterliegen ebenfalls der genannten Creative Commons Lizenz, sofern sich aus der Abbildungslegende nichts anderes ergibt. Sofern das betreffende Material nicht unter der genannten Creative Commons Lizenz steht und die betreffende Handlung nicht nach gesetzlichen Vorschriften erlaubt ist, ist für die oben aufgeführten Weiterverwendungen des Materials die Einwilligung des jeweiligen Rechteinhabers einzuholen.

Weitere Details zur Lizenz entnehmen Sie bitte der Lizenzinformation auf http://creativecommons.org/ licenses/by/4.0/deed.de.

Interessenkonflikt L. Rose gibt an, dass kein Interessenkonflikt besteht.

\section{Literatur}

Althusser, Louis. 1977. Ideologie und ideologische Staatsapparate. Aufsätze zur marxistischen Theorie. Hamburg: Verlag für das Studium der Arbeiterbewegung.

Beck-Gernsheim, Elisabeth. 1990. Alles aus Liebe zum Kind. In: Das ganz normale Chaos der Liebe, Hrsg. U. Beck, und E. Beck-Gernsheim. 135-182, Berlin: Suhrkamp.

Bröckling, Ulrich. 2002. Das unternehmerische Selbst und seine Geschlechter. Leviathan 30(2):175-194.

Cliff, Dallas, und Ruth Deery. 1996. Too much like school: social class, age, marital status and attendance/non-attendance at antenatal classes. Midwifery 13:139-145.

Dackweiler, Regina-Maria. 2010. Frauen(gesundheits)bewegung im Wohlfahrtsstaat: Von der Selbstbestimmung zur "Selbstverantwortung“ - von der Autonomie zur „Selbststeuerung“? In Frauengesundheit in Theorie und Praxis. Feministische Perspektiven in den Gesundheitswissenschaften, Hrsg. G. Mauerer, 145-165. Bielefeld: transcript.

Davis-Floyd, Robbie. 1992. Birth as an American rite of passage. Comparative studies of health systems and medical care. Berkeley: University of California Press.

Dierks, Marie-Luise, Eva-Maria Bitzer, Magnus Lerch, Sabine Martin, Sabine Röseler, Anja Schienkiewitz, und Stefanie Siebeneick. 2001. Patientensouveränität - Der autonome Patient im Mittelpunkt. Arbeitsbericht Nr. 195. Stuttgart: Akademie für Technikfolgenabschätzung in Baden-Württemberg.

Forsa. 2013. Meinungen und Einstellungen der Väter in Deutschland. https://docplayer.org/11610578Meinungen-und-einstellungen-der-vaeter-in-deutschland.html. Zugegriffen: 11. Apr. 2021.

Hardach-Pinke, Irene. 1982. Schwangerschaft und Identität. In Die Wiederkehr des Körpers, Hrsg. D. Kamper, C. Wulf, 193-208. Frankfurt am Main: Suhrkamp.

Heimerdinger, Timo. 2012. Simply the Best. Elternschaft als kompetitive Praxis. In Kulturen des Wettbewerbs. Formationen kompetitiver Logiken, Bd. 10, Hrsg. M. Tauschek, 249-267. Münster: Waxmann.

Heimerl, Birgit. 2013. Die Ultraschallsprechstunde. Eine Ethnografie pränataldiagnostischer Situationen. Bielefeld: transcript.

Heimerl, Birgit, und Peter Hofmann. 2016. Wie konzipieren wir Kinderkriegen? Normativer Rationalismus versus empirische Praxisforschung. Zeitschrift für Soziologie 6:410-430.

Jung, Tina. 2017. Die ,gute Geburt“ - Ergebnis richtiger Entscheidungen? Zur Kritik des gegenwärtigen Selbstbestimmungsdiskurses vor dem Hintergrund der Ökonomisierung des Geburtshilfesystems. GENDER. Zeitschrift für Geschlecht, Kultur und Gesellschaft 2:30-45. 
Kelle, Helga. 2004. Ethnographische Ansätze in der erziehungswissenschaftlichen Frauen- und Geschlechterforschung. In Handbuch Gender und Erziehungswissenschaft, Hrsg. E. Glaser, et al., 636-650. Bad Heilbrunn: Klinkhardt.

Krahl, Astrid. 2012. Aktueller Forschungsstand zur Bedeutung der Geburtsvorbereitung. In Geburtsvorbereitung. Kurskonzepte zum Kombinieren, Hrsg. Deutscher Hebammenverband, 2-9. Stuttgart: Hippokrates.

Krumbügel, Janne. 2015. Der Übergang zur Elternschaft in ärztlichen Schwangerschaftsratgebern. Eine diskursanalytische Untersuchung von Mutter- und Vaterbildern. In Mutter, Vater, Kind - Geschlechterpraxen in der Elternschaft, Hrsg. R. Seehaus, et al., 141-156. Opladen: Barbara Budrich.

Lange, Ute, und Charlotte Ullrich. 2018. Schwangerschaft und Geburt: Perspektiven und Studien aus der Geschlechterforschung. In Handbuch Interdisziplinäre Geschlechterforschung. Geschlecht und Gesellschaft, Hrsg. B. Kortendiek, et al. Wiesbaden: VS. https://doi.org/10.1007/978-3-658-12500-4_ 74-2.

Macdonald, Margaret. 2006. Gender expectations: natural bodies and natural births in the new midwifery in Canada. Medical Anthropology Quarterly 20:235-256.

Metz-Becker, Marita. 1997. Der verwaltete Körper: Die Medikalisierung schwangerer Frauen in den Gebärhäusern des frühen 19. Jahrhunderts. Frankfurt am Main: Campus.

Müller, Marion, und Nicole Zillien. 2016. Das Rätsel der Retraditionalisierung - Zur Verweiblichung von Elternschaft in Geburtsvorbereitungskursen. Kölner Zeitschrift für Soziologie und Sozialpsychologie https://doi.org/10.1007/s11577-016-0374-5.

Neumann, Regina, und Adelheid Smolka. 2016. Familienbildung aus Sicht Bayerischer Mütter und Väter. Ergebnisse der dritten ifb-Elternbefragung zur Familienbildung. Staatsinstitut für Familienforschung an der Universität Bamberg (ifb).

Oakley, Ann. 1980. Women confined: towards a sociology of childbirth. Oxford: Robertson.

Oelkers, Nina. 2007. Aktivierung von Elternverantwortung: Zur Aufgabenwahrnehmung in Jugendämtern nach dem neuen Kindschaftsrecht. Bielefeld: transcript.

Rafalovich, Adam. 2016. Pain is the club: identity and membership in the natural childbirth community. Qualitative Sociology Review 12(3):100-116.

Rieger, Stefan. 2002. Arbeit an sich. Dispositive der Selbstsorge in der Moderne. In Anthropologie der Arbeit, Hrsg. U. Bröckling, E. Horn, 79-96. Tübingen: Narr.

Rose, Lotte. 1993. Kinderkriegen heute. Riskante Chancen zwischen Apparatemedizin und „sanfter“ Geburtskultur. In Was für Kinder. Aufwachsen in Deutschland. Ein Handbuch, Hrsg. Deutsches Jugendinstitut, 95-100. München: Kösel.

Rose, Lotte, und Judith Pape. 2020. Geschlechterordnungen der Familie. Ethnografische Eindrücke zur Positionierung des Vaters in Bildungsangeboten zur Geburt und ersten Lebenszeit des Kindes. In Sozialarbeitswissenschaftliche Geschlechterforschung. Methodologische Fragen, Forschungsfelder und empirische Erträge, Hrsg. L. Rose, E. Schimpf, 183-200. Opladen, Berlin, Toronto: Barbara Budrich.

Rose, Lotte, und Birgit Planitz. 2021. Der ungleiche Start ins Leben. Soziale Differenzen ,rund um die Geburt" als wissenschaftliche und sozialpolitische Herausforderung. In Gelingende Geburt, Hrsg. O. Mitscherlich-Schönherr, R. Anselm, 247-269. Berlin, Boston: de Gruyter.

Ruhl, Lealle. 1999. Liberal governance and prenatal care: risk and regulation in pregnancy. Economy and Society 28(1):95-117.

Schäfer, Eberhard. 2009. Familienbildung muss vätergerecht werden. In Väterarbeit in Niedersachsen, Hrsg. Niedersächsisches Ministerium für Soziales, Frauen, Familie und Gesundheit, 39-42. Hannover: Eigenverlag.

Schirrmacher, Gesa. 2009. Väter in der (Familien-)Bildung. In Väterarbeit in Niedersachsen, Hrsg. Niedersächsisches Ministerium für Soziales, Frauen, Familie und Gesundheit, 37-38. Hannover: Eigenverlag.

Schwarz, Clarissa, und Beate Schücking. 2004. Adieu, normale Geburt? Dr. med Mabuse 148:22-25.

Strauss, Anselm, und Juliet Corbin. 1996. Grounded theory. Grundlagen qualitativer Sozialforschung. Weinheim: Beltz.

Süddeutsche Zeitung. 2014. 95 Prozent der neuen Väter bei Geburt dabei (13. Januar 2014). www. sueddeutsche.de/leben/familie-95-prozent-der-neuen-vaeter-bei-geburt-dabei-dpa.urn-newsmldpa-com-20090101-140113-99-06104?print=true. Zugegriffen: 9. Okt. 2019.

Tews, Marjorie. 2007. Sichere Geburt? Eine kritische Auseinandersetzung mit der Geschichte der Geburtshilfe. Frankfurt am Main: Mabuse.

Villa, Paula Irene, Stephan Moebius, und Barbara Thiessen (Hrsg.). 2011. Soziologie der Geburt: Diskurse, Praktiken und Perspektiven. Frankfurt am Main, New York: Campus. 
Völkle, Laura, und Eva Muthmann. 2019. „Natürliche“ Kompetenzen in der Krise - Konstruktionen von Unfähigkeit beim Gebären und Stillen. Österreichische Zeitschrift für Soziologie 3:333-349. https:// doi.org/10.1007/s11614-019-00363-2.

Lotte Rose ist Diplom-Pädagogin und hat in den Erziehungswissenschaften promoviert. Seit 1997 ist sie Professorin an der Frankfurt University of Applied Sciences, Fachbereich Soziale Arbeit und Leitung des Gender- und Frauenforschungszentrums der Hessischen Hochschulen (gFFZ). Ihre Forschungsschwerpunkte sind Gender Studies, Food Studies, Fat Studies, Human Animal Studies und Elternschaftsforschung. 